\title{
Megastudie stellt Ernährungsempfehlungen infrage
}

\section{Eine fettarme Ernährung ist nach den Ergebnissen einer großen Ernährungsstudie nicht mehr zu empfehlen.}

— Für die PURE-Studie wurden 135.335 Menschen aus 18 Ländern zu ihren Ernährungsgewohnheiten befragt und über 7,4 Jahre nachbeobachtet.

Dabei stellte sich heraus, dass diejenigen, die sich fettreich ernährten, deutlich länger lebten als jene mit einer niedrigen Fettzufuhr (höchstes vs. niedrigstes Quintil). Die Gesamtmortalität lag signifikant um 23\% niedriger. Selbst ein erhöhter Konsum von gesättigten Fettsäuren ging mit einer 14\% geringeren Sterblichkeit einher, bei einfach gesättigten Fettsäuren war diese um 19\% und bei mehrfach gesättigten Fettsäuren um $20 \%$ geringer. Eine hohe Zufuhr von Kohlenhydraten ging dagegen mit einem um $28 \%$ erhöhten Sterberisiko einher.

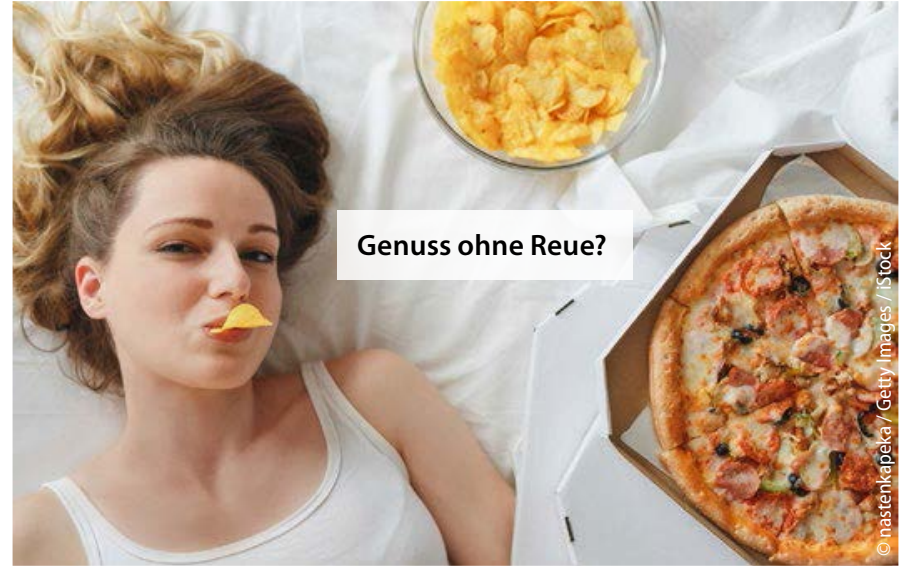

Obst, Gemüse und Hülsenfrüchte: Drei bis vier Portionen genügen In der PURE-Studie wurde auch der Zusammenhang zwischen dem Konsum von Obst, Gemüse und Hülsenfrüchten und der Mortalität untersucht: Bei drei bis vier Portionen pro Tag war das Sterberisiko um $22 \%$ geringer als bei weniger als einer Portion. Eine noch höhere Zufuhr (die Leitlinien empfehlen derzeit noch mindes-

Dr. Andrew Mente von der McMaster University in Hamilton hält die aktuellen Ernährungsempfehlungen aufgrund dieser Ergebnisse für fragwürdig: „Wir sollten uns von der Fettrestriktion lösen.“ Menschen mit einem hohen Kohlenhydratanteil in der Ernährung (> 60\%) sollten diesen auf ein gemäßigtes Maß reduzieren. Von einem hohen Fettanteil gehe dagegen keine Gefahr aus. Von fettreduzierten Lebensmitteln rät Mente ab. tens fünf Portionen am Tag) brachte keinen weiteren Zugewinn an Lebenszeit.

Trotz ihrer Größe ist diese Studie nicht ohne Limitationen. Die Angaben zur Ernährung wurden nur zu Beginn erfasst. Angaben zur Zubereitungsweise der Nahrungsmittel fehlen, und der Anteil der als besonders schädlich geltenden Transfette wurde nicht erfasst.

Veronika Schlimpert

- ESC-Kongress, 29.8.2017 in Barcelona

\section{DETO2X-AMI-Studie}

\section{Herzinfarkt: Keine routinemäßige $0_{2}$-Gabe mehr?}

\section{Die Zufuhr von Sauerstoff ist bei Pati- enten mit Verdacht auf Herzinfarkt eine häufige Routinemaßnahme - auch dann, wenn keine Hypoxie besteht. Künftig kann darauf in solchen Fällen wohl getrost verzichtet werden.}

— In der DETO2X-AMI-Studie wurde die Überlebensrate von normoxämischen Infarktpatienten durch Sauerstoffgabe nicht verbessert. Auch auf Reinfarkte und die anhand von Biomarkern (Troponine) objektivierbare Myokardschädigung hatte sie keinen Einfluss.

In die Studie wurden 6.229 Patienten mit Anzeichen für einen akuten Herzin- farkt und einer Sauerstoffsättigung im Blut von $>90 \%$ aufgenommen. Eine Hälfte bekam über eine Atemmaske knapp 12 Stunden lang Sauerstoff zugeführt, die andere Hälfte atmete nur Raumluft ohne Maske ein. Am Ende dieser Phase betrug die Sauerstoffsättigung in den Gruppen mit und ohne Sauerstoffgabe 99 bzw. 97\%.

\section{Kein Unterschied bei der 1-Jahres-Mortalität}

Nach einem Jahr waren die Mortalitätsraten mit 5,0\% (Sauerstoff-Gruppe) und $5,1 \%$ (Kontrollgruppe) nahezu identisch. Die Raten für die in dieser Zeit registrierten erneuten Klinikeinweisungen wegen
Herzinfarkt waren ebenfalls kaum unterschiedlich $(3,8$ vs. $3,3 \%)$. Und auch bei Subgruppen von Hochrisikopatienten wie Raucher, ältere Patienten sowie Patienten mit Diabetes oder vorbestehender Herzerkrankung waren keine Unterschiede bezüglich der Sterberisikos auszumachen.

„Die Studie wird wahrscheinlich unmittelbare Auswirkungen auf die Praxis und künftige Leitlinien haben“, prognostiziert Studienautor Prof. Stefan James von der Universität Uppsala.

Peter Overbeck

- Kongress der European Society of Cardiology (ESC) 2017, 29.-30. August 2017, Barcelona

Hofman R et al. N Engl J Med, online 28. August 2017, https://doi.org/10.1056/NEJMoa1706222 